\title{
VALUE OF SERUM MIDKINE LEVEL IN PATIENTS WITH LIVER CIRRHOSIS AND HEPATOCELLULAR CARCINOMA
}

\author{
By
Ahmed Ibrahim El-Said Mandour, Magdy Abd El-Karem Al-Dahshan, Omar Mohamed Khalil Heikal, Mohamed Saad El-Din Radwan* and Mohamed Shahat Hasib El-Fayoumie
Departments of Internal Medicine and Clinical Pathology*, Faculty of Medicine, Al-Azhar University

Corresponding author: Ahmed Ibrahim El Said Mandour;

E-mail: ahmedmandour24@gmail.com

\begin{abstract}
Background: Hepatocellular carcinoma (HCC) is the fourth most common cause of cancer-related death worldwide. Risk factors for HCC include chronic hepatitis B and hepatitis C, alcohol addiction, metabolic liver disease and exposure to dietary toxins such as aflatoxins and aristolochic acid.

Objective: To determine the value of serum Medkine level as a potential biomarker for patients with hepatocellular carcinoma.

Patients and methods: This study was conducted at the Gastroenterology and Hepatology Unit, Department of Internal Medicine Al-Azhar and Misr University for Science and Technology. 90 Egyptian patients were recruited and were divided into 3 Group I included 30 patients with liver cirrhosis, HCC was excluded in these patients at time of recruitment in the study, exclusion of HCC was based on the absence of any hepatic focal lesion in abdominal ultrasonography scanning. Group II included 40 patients with HCC. Diagnosis of $\mathrm{HCC}$ was based on the appearance of typical vascular pattern of enhancement in triphasic spiral CT scan of the abdomen. Control Group included 20 age and sex-matched apparently healthy subjects.

Results: Different liver function tests showed no statistically difference between two groups except for ALK. $\mathrm{Ph}$ and gamma glutamyltransferase (GGT) which were significant higher in group II than group I. There was a highly statistically significance difference regarding alpha-fetoprotein (AFP) which was higher in group II than group I and control group. Serum AFP levels were found to be significantly correlated with larger tumor size in this study. In addition, patients with advanced-stage hepatocellular carcinomas had significantly higher median AFP serum levels (BCLC B/C) than that of early-stage tumors (BCLC 0/A) (237ng/mL versus $23 \mathrm{ng} / \mathrm{mL}$ ). However, no significant correlation was found between serum midkine (MDK) levels with tumor size, number or serum levels of AFP, and no significant association was found between serum MDK levels and BCLC stages. The best cutoff values for MDK and AFP to discriminate HCC cases from those with liver cirrhosis were 0.39 and $10 \mathrm{ng} / \mathrm{mL}$, respectively, with sensitivities $(90 \%$ versus $77.5 \%)$, specificities $(60 \%$ versus $80 \%$ ). The overall diagnostic performance of MDK for HCC diagnosis was much better than that of AFP. On comparing the sensitivities and specificities of MDK at the cutoff $0.39 \mathrm{ng} / \mathrm{mL}$ to those of AFP at different cutoff values (10 and 400), the overall diagnostic performance of MDK for HCC diagnosis was much better than that of AFP.
\end{abstract}

Conclusion: Alpha-fetoprotein and midkine may have a complementary role in hepatocellular carcinoma surveillance and screening. Midkine increased the diagnostic yield in alpha-fetoprotein -negative hepatocellular carcinoma and the presence of either elevated alpha-fetoprotein or midkine increased the sensitivity of hepatocellular carcinoma detection. Midkine was also superior to alpha-fetoprotein in the 
diagnosis of NASH-related hepatocellular carcinoma and this finding postulated an exciting novel role for midkine in NASH-related carcinogenesis.

Keywords: Serum Midkine Level, Liver Cirrhosis, Hepatocellular Carcinoma.

\section{INTRODUCTION}

Hepatocellular carcinoma (HCC) is a common and deadly malignancy. The disease usually develops on a background of chronic liver disease. The most common etiology was infection with the hepatitis $\mathrm{C}$ virus (HCV). The advent of direct-acting antiviral (DAA) therapies has been a major breakthrough in $\mathrm{HCV}$ treatment. Sustained virologic response can now be achieved in almost all treated patients, even in patients with a high risk for the development of HCC, such as the elderly or those with significant fibrosis. Simultaneous to the decrease in the burden of cirrhosis from $\mathrm{HCV}$, nonalcoholic fatty liver disease (NAFLD) incidence has been increasing dramatically including significant increased incidence of cirrhosis and HCC in these patients. Surprisingly, a substantial proportion of patients with NAFLD were shown to develop HCC even in the absence of cirrhosis. Furthermore, HCC treatment and potential complications are known to be influenced by liver steatosis (Meringer et al., 2019).

Information about the incidence of HCC in patients with nonviral chronic liver disease without cirrhosis, such as alcoholic and non-alcoholic steatohepatitis (NASH), autoimmune liver disease, genetic hemochromatosis, alpha-1antitrypsin deficiency, and Wilson's disease are limited. However, available evidence suggests that HCC usually arises in these contexts once cirrhosis is established (Forner et al., 2018).
One reason for the lack of a single specific and sensitive HCC biomarker is the highly heterogeneous nature of HCC at a molecular level not only between individuals, but between tumors within the one individual, and even within single tumors (Giannelli et al., 2014). A significant limitation to the use of AFP for HCC surveillance is the rate of AFPnegative HCC. Up to $50 \%$ of small HCCs do not secrete AFP and even with larger lesions, $20 \%$ are not associated with elevated levels (Sherman et al., 2014).

Hence, single biomarkers are unlikely to capture the complexity of pathways driving hepatocarcinogenesis. Consequently, a paradigm shift from searching for the single biomarker, to profiling a combination of biomarkers might be required to maximize diagnostic yield (Bruix et al., 2014).

Midkine (MK) is a heparin-binding growth factor that markedly expressed during embryogenesis but down regulated to inconsiderable levels in healthy adults. However, MK is up regulated during tissue repair and in many pathologic conditions, mostly malignancies and inflammatory diseases. MK promotes a number of functions in target cells such as migration, proliferation, survival, growth, reproduction and repair, angiogenesis, and gene expression. It acts as a proinflammatory cytokine and contributes to chronic inflammation via promoting chemotaxis and tissue infiltration of neutrophils and macrophages. Furthermore, MK up regulated the 
production of various inflammatory mediators (i.e. interleukin (IL) 6 and IL8) (Aynacıoğlu et al., 2019).

Midkine, a pleiotropic protein and growth factor, has originally been identified to orchestrate embryonic development. In the adult organism, its expression is restricted to distinct tissues (including tumors), whereby midkine are strongly expressed in inflamed tissue and has been shown to promote inflammation. Investigations conferred midkine an important function in vascular remodeling and growth (Weckbach et al., 2018).

Midkine is overexpressed in hepatocellular carcinoma (HCC) and plays a role in tumor progression. Midkine is a basic heparin-binding growth factor that is constitutively active when it forms homodimers that are stabilized by heparin. It is a highly conserved and developmentally regulated gene product, widely expressed in different cell types and strongly induced by retinoic acid during mid-gestation, hence the name "midkine" (Kadomatsu et al., 2013). However, expression of the MDK gene in human adult tissues is extremely low and restricted. Mounting evidence has indicated that MDK plays a significant role in carcinogenesis related activities, such as proliferation, migration, antiapoptosis, mitogenesis, transformation, and angiogenesis, inmany types of solid tumors, including hepatocellular carcinomas (Muramatsu and Kadomatsu, 2014).

Midkine has moderate diagnostic accuracy for HCC. Serum MDK is elevated in most HCC and may have a diagnostic role (Zhang et al., 2020).
In a gene expression profiling study, MDK was identified as one of 5 potential biomarkers for HCC. Another study demonstrated that serum MDK is elevated in most $\mathrm{HCC}$ and may have a diagnostic role in AFP-negative and early stage tumours (Zhu et al., 2013). A higher serum midkine level could be used for detecting early $\mathrm{HCC}$ and predicting metastasis and poor prognosis (Shaheen et al., 2015).

The aim of this study was to determine the value of serum Medkine level as a potential biomarker for patients with hepatocellular carcinoma.

\section{PATIENTS AND METHODS}

This study was conducted at the Gastroenterology and Hepatology Unit, Departments of Internal Medicine AlAzhar and Misr University for Science and Technology.

\section{Ninety Egyptian patients were recruited and were divided into 3 groups as follow:}

Group I: included 30 patients with liver cirrhosis, HCC was excluded in these patients at time of recruitment in the study. Exclusion of HCC was based on the absence of any hepatic focal lesion in abdominal ultrasonography scanning.

Group II: included 40 patients with HCC. Diagnosis of HCC was based on the appearance of typical vascular pattern of enhancement in triphasic spiral CT scan of the abdomen.

Control Group: included 20 age and sexmatched apparently healthy subjects. 
All patients were subjected to the following:

- History taking.

- Thorough clinical examination.

- Laboratory investigations including:

a. Complete blood picture.

b. Liver enzymes and function tests: AST, ALT, serum total proteins, serum albumin, total and direct bilirubin.

c. Prothrombin time and INR.

d. Kidney function tests: BUN, creatinine.

e. Serum alpha feto protein.

f. Serum Midkine level by enzymelinked immunosorbent assay (ELISA).

g. Abdominal ultrasonography.

h. Written informed consent was obtained from every patient before participation in the study.
Patients were excluded in the following cases:

1. Current or past history of any other malignant diseases (solid or humoral).

2. Organ transplant recipients.

3. Patients with HIV infection.

4. Patients refusing to be entitled in the study.

\section{Statistical analysis:}

Recorded data were analyzed using the statistical package for the social sciences, version 20.0 (SPSS Inc., Chicago, Illinois, USA). Quantitative data were expressed as mean \pm standard deviation (SD). Qualitative data were expressed as frequency and percentage. Independentsamples t-test of significance was used when comparing between two means. Chisquare (x2) test of significance was used in order to compare proportions between two qualitative parameters. The confidence interval was set to $95 \%$ and the margin of error accepted was set to $5 \%$. The p-value was considered significant when: P-value $<0.05$.

\section{RESULTS}

Group I included 12 females and 18 males who had median age $50.03 \pm 7.02$ years old and group II involved 15 females and 25 males who had median age $53.45 \pm 8.81$ years. In group I, It was found that the cause of cirrhosis was HCV in 27 patients (90\%), 3 patients had HBV $(10 \%)$, In group II, It was found that the cause of cirrhosis was HCV in 38 patients (95\%), 2 patient had HBV (5\%). The mean age among HCV patients ( group I) patients was $50.3 \pm 7.02$ years, while the mean age among $\mathrm{HCC}$ patients (group II) $(53.45 \pm 8.81$ years $)$. This difference was insignificant (Table 1).

\section{Table (1): Showed the Comparison of age in different groups}

\begin{tabular}{|l|l|c|c|c|c|}
\hline \multirow{2}{*}{ Parmeters } & Groups & Control group & Liver cirrhosis group & HCC group & \multirow{2}{*}{ P-value } \\
\cline { 2 - 5 } Pane & No. $=\mathbf{2 0}$ & $\mathbf{N o .}=\mathbf{3 0}$ & No. $=\mathbf{4 0}$ & \\
\hline \multirow{2}{*}{ Age } & Mean \pm SD & $30.80 \pm 8.01$ & $50.03 \pm 7.02$ & $53.45 \pm 8.81$ & \multirow{2}{*}{0.000} \\
\cline { 2 - 5 } & Range & $19-52$ & $38-63$ & $38-75$ & \\
\hline \multirow{2}{*}{ Sex } & Male & $14(70.0 \%)$ & $18(60.0 \%)$ & $25(62.5 \%)$ & \multirow{2}{*}{0.764} \\
\cline { 2 - 5 } & Female & $6(30.0 \%)$ & $12(40.0 \%)$ & $15(37.5 \%)$ & \\
\hline
\end{tabular}


The value of AFP and MIDKINE were higher among HCC patients (group II) than $\mathrm{HCV}$ patients (group I) and these differences were statistically significant (Table 2).

Table (2): Comparison of basic data between groups

\begin{tabular}{|c|c|c|c|c|c|c|c|}
\hline \multirow{2}{*}{\multicolumn{2}{|c|}{$\mathrm{Farameters}_{\text {Groups }}$}} & \multirow{2}{*}{$\begin{array}{c}\text { Control group } \\
\text { No. }=\mathbf{2 0}\end{array}$} & \multirow{2}{*}{\begin{tabular}{|c|}
$\begin{array}{c}\text { Liver cirrhosis } \\
\text { group }\end{array}$ \\
No. $=\mathbf{3 0}$ \\
\end{tabular}} & \multirow{2}{*}{$\begin{array}{c}\text { HCC group } \\
\text { No. }=\mathbf{4 0}\end{array}$} & \multirow[t]{2}{*}{ Test value } & \multirow[t]{2}{*}{ P-value } & \multirow[t]{2}{*}{ Sig. } \\
\hline & & & & & & & \\
\hline & Negative & $20(100.0 \%)$ & $27(90.0 \%)$ & $38(95.0 \%)$ & & & \\
\hline HBV.Sag & Positive & $0(0.0 \%)$ & $3(10.0 \%)$ & $2(5.0 \%)$ & $2.329^{*}$ & 0.312 & NS \\
\hline & Negative & $20(100.0 \%)$ & $3(10.0 \%)$ & $2(5.0 \%)$ & & & \\
\hline HCV.ab & Positive & $0(0.0 \%)$ & $27(90.0 \%)$ & $38(95.0 \%)$ & $0 / .0 / 1 *$ & 0.000 & HS \\
\hline AFP & Median (IQR) & $2(2-3)$ & $12(7.4-16)$ & $135(9-233.5)$ & & & \\
\hline AFP & Range & $1-6$ & $2-58$ & $6-11364$ & 14. & 0.000 & HS \\
\hline & Mean \pm SD & $0.12 \pm 0.01$ & $0.28 \pm 0.20$ & $0.68 \pm 0.16$ & & & \\
\hline IMIDKINE & Range & $0.1-0.14$ & $0.14-0.74$ & $0.34-1.23$ & $0^{\bullet}$ & 0 & HS \\
\hline & A & - & $9(30.0 \%)$ & $15(37.5 \%)$ & & & \\
\hline CHILD & $\mathrm{B}$ & - & $8(26.7 \%)$ & $10(25.0 \%)$ & $0.446^{*}$ & 0.800 & NS \\
\hline & $\mathrm{C}$ & - & $13(43.3 \%)$ & $15(37.5 \%)$ & & & \\
\hline & & & Post hoc anal & & & & \\
\hline & Control Vs li & iver cirrhosis & Control & s HCC & Liv & hosis V & $\overline{C C}$ \\
\hline AFP & 0.0 & 000 & 0.0 & & & .000 & \\
\hline MIDKINE & 0.0 & 001 & 0.0 & & & .000 & \\
\hline
\end{tabular}

There was a significant positive correlation between AFP and (midkine, number of focal lesion, size of focal lesion, GGT and ALK.P) in HCC group II on the other hand significant negative correlation between AFP and Na in HCC group II (Table 3).

Table (3): Correlation between AFP and (laboratory and basic) data in the whole study subjects

\begin{tabular}{|l|c|c|c|c|c|c|}
\hline \multirow{2}{*}{ Parameters } & \multicolumn{2}{|c|}{ All patients group } & \multicolumn{2}{c|}{ Liver cirrhosis group } & \multicolumn{2}{c|}{ HCC group } \\
\cline { 2 - 7 } & $\mathbf{r}$ & P-value & R & P-value & r & P-value \\
\hline MIDKINE & $0.601 * *$ & 0.000 & 0.054 & 0.778 & $0.730^{* *}$ & $<0.001$ \\
\hline Age & -0.021 & 0.864 & -0.328 & 0.077 & -0.064 & 0.694 \\
\hline Hb & -0.039 & 0.750 & 0.168 & 0.375 & -0.027 & 0.870 \\
\hline PLT & 0.036 & 0.768 & 0.217 & 0.249 & 0.105 & 0.518 \\
\hline WBC & -0.213 & 0.076 & -0.091 & 0.632 & -0.195 & 0.227 \\
\hline ALT & -0.195 & 0.106 & -0.127 & 0.505 & -0.189 & 0.242 \\
\hline AST & -0.073 & 0.551 & -0.065 & 0.733 & -0.080 & 0.622 \\
\hline ALK.P (+ve) & $.447 * *$ & 0.000 & -0.324 & 0.080 & $0.730 * *$ & 0.000 \\
\hline GGT $(+$ ve) & $.511 * *$ & 0.000 & -0.113 & 0.551 & $0.626 * *$ & $<0.001$ \\
\hline S.ALB & 0.063 & 0.604 & -0.086 & 0.650 & 0.197 & 0.223 \\
\hline T.P & $-0.318 * *$ & 0.007 & -0.301 & 0.106 & -0.308 & 0.053 \\
\hline PT & 0.085 & 0.487 & 0.052 & 0.787 & 0.115 & 0.481 \\
\hline INR & -0.027 & 0.827 & -0.178 & 0.345 & 0.113 & 0.489 \\
\hline T.B & 0.020 & 0.870 & -0.051 & 0.791 & 0.070 & 0.668 \\
\hline D.B & -0.046 & 0.707 & -0.011 & 0.954 & -0.026 & 0.874 \\
\hline S.CRT & -0.027 & 0.826 & $-0.369 *$ & 0.045 & 0.191 & 0.237 \\
\hline UREA & -0.052 & 0.671 & -0.172 & 0.362 & -0.060 & 0.715 \\
\hline NA & -0.198 & 0.100 & 0.073 & 0.701 & $-0.438 * *$ & 0.005 \\
\hline K & -0.115 & 0.345 & -0.005 & 0.978 & -0.079 & 0.628 \\
\hline NO.of FL. & $0.521 * *$ & 0.001 & & & $0.521 * *$ & 0.001 \\
\hline Size of FL & $0.484 * *$ & 0.002 & & & $0.484 * *$ & 0.002 \\
\hline
\end{tabular}


There was a significant positive correlation between Midkine and (AFP,

GGT and ALK.P) in HCC group II (Table 4).

Table (4): Showed the correlation between Midkine and (laboratory and basic) data in the whole study subjects, cirrhotic patients without HCC (group I), cirrhotic patients with HCC (group II) and control group

\begin{tabular}{|l|c|c|c|c|c|c|}
\hline \multirow{2}{*}{ Parameters } & \multicolumn{2}{c|}{ MIDKINE } & All patients group & \multicolumn{2}{c|}{ Liver cirrhosis group } & \multicolumn{2}{c|}{ HCC group } \\
\cline { 2 - 7 } & $\mathbf{r}$ & P-value & R & P-value & r & P-value \\
\hline AFP (+ve) & $0.601^{* *}$ & 0.000 & 0.054 & 0.778 & $0.730^{* *}$ & $<0.001$ \\
\hline Age & 0.186 & 0.124 & -0.255 & 0.173 & 0.226 & 0.161 \\
\hline Hb & -0.141 & 0.243 & -0.360 & 0.051 & 0.019 & 0.907 \\
\hline PLT & -0.184 & 0.128 & -0.073 & 0.703 & 0.014 & 0.933 \\
\hline WBC & -0.089 & 0.464 & 0.120 & 0.528 & -0.124 & 0.445 \\
\hline ALT & 0.034 & 0.779 & 0.349 & 0.059 & 0.058 & 0.721 \\
\hline AST & 0.096 & 0.428 & 0.235 & 0.211 & -0.005 & 0.974 \\
\hline ALK.P $(+v e)$ & $0.466^{* *}$ & 0.000 & -0.046 & 0.810 & $0.652^{* *}$ & $<0.001$ \\
\hline GGT $(+v e)$ & $0.478^{* *}$ & 0.000 & -0.179 & 0.344 & $0.496^{* *}$ & 0.001 \\
\hline S.ALB & -0.017 & 0.891 & 0.335 & 0.071 & -0.213 & 0.187 \\
\hline T.P & 0.077 & 0.526 & 0.329 & 0.076 & 0.143 & 0.379 \\
\hline PT & 0.155 & 0.199 & $0.373^{*}$ & 0.042 & 0.122 & 0.452 \\
\hline INR & 0.071 & 0.558 & 0.315 & 0.090 & 0.140 & 0.391 \\
\hline T.B & 0.062 & 0.612 & 0.180 & 0.342 & -0.030 & 0.853 \\
\hline D.B & -0.008 & 0.947 & 0.235 & 0.211 & -0.095 & 0.558 \\
\hline S.CRT & 0.020 & 0.871 & 0.138 & 0.467 & 0.028 & 0.862 \\
\hline UREA & 0.057 & 0.637 & -0.026 & 0.892 & -0.044 & 0.790 \\
\hline NA & -0.051 & 0.674 & 0.127 & 0.504 & $-0.370^{*}$ & 0.019 \\
\hline K & -0.170 & 0.161 & 0.227 & 0.228 & -0.156 & 0.337 \\
\hline NO.of FL. & 0.240 & 0.135 & & & 0.240 & 0.135 \\
\hline Size of FL & 0.080 & 0.625 & & & 0.080 & 0.625 \\
\hline
\end{tabular}

This table shows that AFP cutoff value $>16$ has $77.5 \%$ sensitivity, $80 \%$ specificity $83.7 \%$ PPV and $72.7 \%$ NPV in detecting $\mathrm{HCC}$ patients and Midkine cutoff value $>0.37$ has $90 \%$ sensitivity, $60 \%$ specificity $75 \%$ PPV and $81.8 \%$ NPV (Table 5\& Figure 1). 


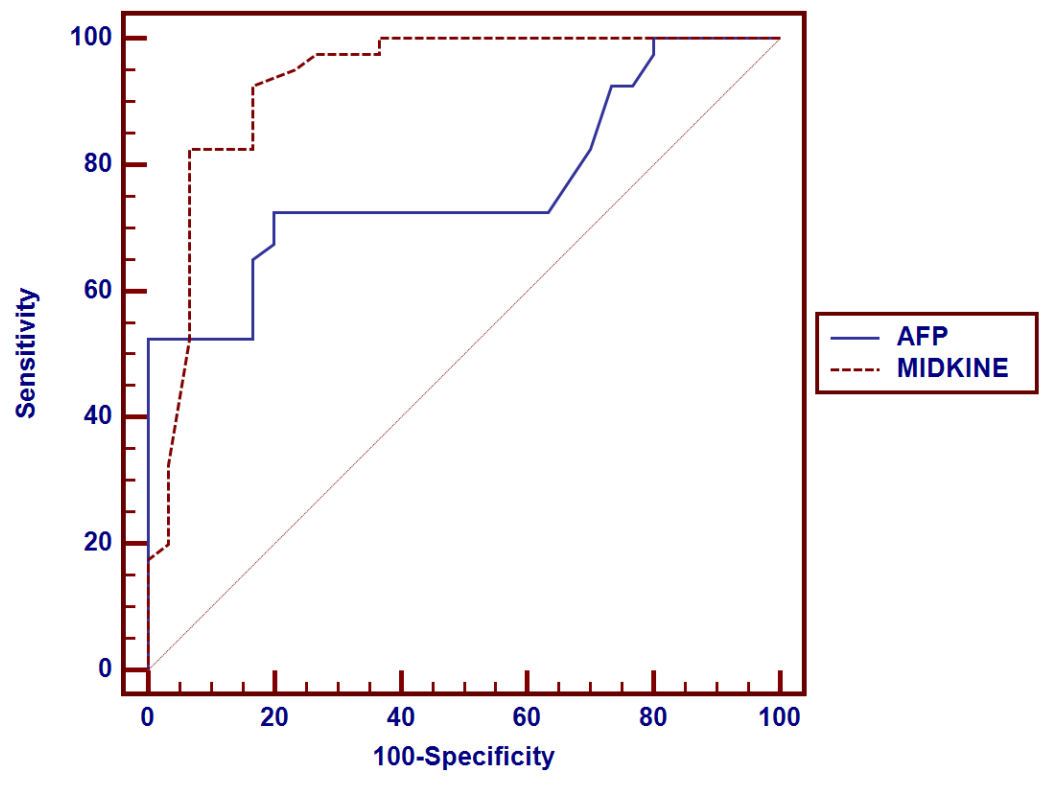

\begin{tabular}{|c|c|c|c|c|c|c|}
\hline Variables & Cut off point & AUC & Sensitivity & Specificity & +PV & -PV \\
\hline AFP & $>16$ & 0.767 & $77.5 \%$ & 80.0 & $83.7 \%$ & $72.7 \%$ \\
\hline MIDKINE & $>0.37$ & 0.928 & $90 \%$ & $60 \%$ & $75 \%$ & $81.8 \%$ \\
\hline
\end{tabular}

Figure (1): ROC curve of AFP and MEDKIN between liver cirrhosis group and HCC group to predict $\mathrm{HCC}$ group

To discriminate patients with HCC and those with LC, the sensitivity of MDK at cutoff value $>0.39 \mathrm{ng} / \mathrm{mL}$ was found to be much significantly higher when compared to that of AFP at cutoff values $>10$, and $400 \mathrm{ng} / \mathrm{mL}$ (90\% versus $77.5 \%$ and $57 \%$, respectively.), while the specificity of
MDK cutoff value $>0.39 \mathrm{ng} / \mathrm{mL}$ was $60 \%$ of versus AFP at cutoff value 20 and 400 $\mathrm{ng} / \mathrm{mL}(80 \%$ and $100 \%)$. Both of MDK and AFP had excellent diagnostic performance to diagnose and differentiate HCC group with sensitivity $100 \%$ and specificity $34 \%$.

Table (5): Comparison between MDK at cutoff value $0.37 \mathrm{ng} / \mathrm{mL}$ and AFP at cutoff values $16 \mathrm{ng} / \mathrm{mL}$ and $400 \mathrm{ng} / \mathrm{mL}$ regarding sensitivity and specificity in diagnosis of $\mathrm{HCC}$

\begin{tabular}{|l|c|c|c|c|}
\hline Diagnosis & Sensitivity & Specificity & PPV & NPV \\
\hline AFP > 16 ng/ml & $77.5 \%$ & $80 \%$ & $83.7 \%$ & $72.7 \%$ \\
\hline MIDKINE >0.37 ng/ml & $90 \%$ & $60 \%$ & $75 \%$ & $81.8 \%$ \\
\hline AFP > 16 ng/ml+ MIDKINE >0.37 ng/ml* & $100 \%$ & $33.3 \%$ & $71.4 \%$ & $100 \%$ \\
\hline AFP > 16 ng/ml+ MIDKINE >0.37 ng/ml\# & $77.5 \%$ & $90 \%$ & $91.1 \%$ & $75 \%$ \\
\hline AFP > 400 ng/ml & $57 \%$ & $100 \%$ & $100 \%$ & $63 \%$ \\
\hline MIDKINE >0.37 ng/ml & $90 \%$ & $60 \%$ & $75 \%$ & $81.8 \%$ \\
\hline AFP > 400 ng/ml+ MIDKINE >0.37 ng/ml* & $100 \%$ & $33.3 \%$ & $71.4 \%$ & $100 \%$ \\
\hline AFP > 400 ng/ml+ MIDKINE >0.37 $\mathbf{~ n g / m l ~}$ & $55 \%$ & $100 \%$ & $100 \%$ & $62 \%$ \\
\hline
\end{tabular}

* The result of test was considered positive if one of both tests was positive.

\# The result was considered positive provided that both of tests were positive. 


\section{AHMED IBRAHIM MANDOUR et al.,}

\section{DISCUSSION}

In Egypt, a large study, evaluated the epidemiological characteristics of $\mathrm{HCC}$, stated that $\mathrm{HCV}$ is the predominant cause of the underlying liver cirrhosis constituting about $91.32 \%$ of $\mathrm{HCC}$ cases, while chronic HVB infection was reported in $2.51 \%$ (Shaker et al., 2013). This is very close to our results where $97 \%$ of HCC cases had chronic HCV and only 5\% had chronic HBV infection. When used as a diagnostic test, AFP levels at a value of $10 \mathrm{ng} / \mathrm{mL}$ show low specificity but fair sensitivity $(77.5 \%)$; that is, AFP surveillance would miss $22.5 \%$.

Midkine has an essential role in activities related to carcinogenesis such as proliferation, anti-apoptosis, transformation, migration, and angiogenesis, in many types of tumors, including HCC (Shaheen et al., 2015). Midkine may serves as a novel diagnostic marker for HCC with better sensitivity and accuracy than AFP. The combined determination of midkine and AFP increase the sensitivity of HCC detection (Mao et al., 2010).

The present study determined the value of serum midkine level in patients with liver cirrhosis and HCC to correlate these levels in prediction and diagnosis of HCC.

In the present study, the mean age among patients with HCV (group A) was $50.03 \pm 7.02$ year, while the mean age among patients with HCC (group B) was $53.45 \pm 8.81$ years with insignificant difference between both groups. Opposite to these results, Moucari et al. (2010) reported that most of HCC patients were over 57 years and the number in this age group showed significant difference when compared to $\mathrm{CHC}$ patients. In the present study, there was no statistically difference between two groups as gender, CBC parameters except platelets count that was statistically significant lower in group II than group I. This could be explained by pathogenesis of thrombocytopenia is multifactorial. Possible causes include splenic sequestration of platelets, suppression of platelet production in the bone marrow, and decreased activity of the hematopoietic growth factor thrombopoietin, also other factors are involved. Increased destruction of platelets within the spleen, intrasplenic production of autoantibodies, and plasma expansion resulting in hemodilution can also contribute to thrombocytopenia (Chan et al., 2017).

Different liver function tests showed no statistical difference between two groups except for ALK. $\mathrm{Ph}$ and GGT which were significant higher in group II than group I. The activity of serum GGT is extremely low in healthy adults, but stimulations such as cholestasis and inflammation can improve the level of serum GGT to varying degrees. GGT mRNA is widely distributed in the liver tissues of healthy adults, patients with liver disease, benign liver tumor and HCC (Singhal et al., 2012).

The present study also demonstrated that there was a statistically significance difference regarding AFP which was higher in group II than group I and control group. Serum AFP levels were found to be significantly correlated with larger tumor size in this study. In addition, patients with advanced-stage hepatocellular carcinomas had significantly higher median AFP serum levels (BCLC B/C) than that of early-stage tumors. However, 
no significant correlation was found between serum MDK levels with tumor size, number or serum levels of AFP, and no significant association was found between serum MDK levels and BCLC stages. These results were in agreement with Stefaniuk et al. (2010) has confirmed that AFP serum concentration increases in parallel with HCC tumor size. For this reason, AFP has to be considered ,the golden standarde for HCC serum markers.

In this study, we found that serum MDK significantly elevated in patients with hepatocellular carcinomas compared with liver cirrhosis patients versus $0.28 \pm$ $0.20 \mathrm{ng} / \mathrm{ml}$ ) and the healthy controls versus $0.12 \pm 0.01$ In addition, serum MDK was not significantly higher in the liver cirrhosis group than that in healthy group, in contrast to serum AFP which significantly elevated in the liver cirrhosis group when compared to healthy group. So, the well-known nonspecific elevations of AFP in patients with liver cirrhosis were not significantly elicited with serum MDK increasing its specificity as a novel diagnostic marker for HCC.

The best cutoff values for MDK and AFP to discriminate HCC cases from those with liver cirrhosis were 0.39 and $10 \mathrm{ng} / \mathrm{mL}$, respectively, with sensitivities (90\% versus $77.5 \%$ ), specificities $(60 \%$ versus $80 \%$ ). So, the overall diagnostic performance of MDK for HCC diagnosis is much better than that of AFP. At higher cut-offs of $400 \mathrm{ng} / \mathrm{mL}$, the sensitivity drops to $57 \%$ with high specificity. Therefore, reducing the cutoff means that more HCCs would be identified, but at the cost of a progressive increase in the falsepositive rate and this with the agreement (Mehinovic et al., 2018).
Our results also were close to those reported by Zhu et al. (2013) showed that MDK levels significantly elevated in HCC tissues as well as serum samples. Serum MDK at the cutoff value of $0.654 \mathrm{ng} / \mathrm{mL}$ for HCC diagnosis showed an obviously higher sensitivity compared with AFP with similar specificities; even in very early-stage HCC. The sensitivity of MDK significant higher than AFP; in those AFP-negative HCC cases, the sensitivity could reach as high as $89.2 \%$, and serum MDK level was significantly decreased in HCC patients after curative resection and reelevated when tumor relapsed.

On comparing the sensitivities and specificities of MDK at the cutoff $0.39 \mathrm{ng} / \mathrm{mL}$ to those of AFP at different cutoff values (10 and 400), we found that the overall diagnostic performance of MDK for HCC diagnosis was much better than that of AFP. These findings were in agreement with Shaheen et al. (2015) who found that the median values of the MDK levels in the HCC patients were significantly higher than that of cirrhotic patients and the healthy control subjects. Though the median values of MDK levels in the cirrhotic patients were higher than that in the control group, values did not reach significance. They also confirmed that no significant correlation was found between serum MDK levels with tumor size, number or serum levels of AFP. Zhang et al. (2020) analysis revealed that the sensitivity and specificity of $\mathrm{MK}$ for HCC diagnosis were 0.85 (95\% CI 0.780.91 ) and 0.83 (95\% CI 0.76-0.88), respectively. Subgroup analyses showed that MK provided the best efficiency for HCC diagnosis when the cutoff value was greater than $0.5 \mathrm{ng} / \mathrm{mL}$. 
The diagnostic cut-off for MDK was $0.44 \mathrm{ng} / \mathrm{ml}$ (sensitivity $70.9 \%$, specificity $62.2 \%$ ) and for OPN was $33.36 \mathrm{ng} / \mathrm{ml}$ (sensitivity $54.7 \%$, specificity $79.7 \%$ ). To determine whether a combination of biomarkers could improve performance for HCC diagnosis, AFP, MDK and OPN were entered into a binary logistic regression model from which only MDK and AFP were found to be significantly associated with HCC diagnosis and from which the following equation was derived: $3^{*} \log \mathrm{AFP}+\log \mathrm{MDK}$. When this combined score was compared to AFP in HCC diagnosis.

Our results have shown that serum MDK could have a diagnostic role in AFP-negative HCC patients, the study revealed that eight $20 \%$ group II were AFP-negative $\mathrm{HCC}$ at cutoff $(10 \mathrm{IU} / \mathrm{ml})$ and about $75 \%$ had elevated Midkine using the optimal diagnostic cut-off of $0.39 \mathrm{ng} / \mathrm{ml}$ supporting a complementary role of MDK to AFP in HCC diagnosis. These results were in agreement with Hodeib et al. (2017) who had shown the role of serum MDK in AFP-negative was $<20 \mathrm{IU} / \mathrm{ml}$. In patients with HCC, 56.98\% had normal AFP. Of these patients with AFP-negative HCC, $59.18 \%$ had elevated MDK using the optimal diagnostic cut-off of $0.44 \mathrm{ng} / \mathrm{ml}$. Using a criteria of AFP $\geq$ $20 \mathrm{IU} / \mathrm{ml}$ or $\mathrm{MDK} \geq 0.44 \mathrm{ng} / \mathrm{ml}$, a significantly greater number of HCC cases were detected, supporting a complementary role of MDK to AFP in HCC diagnosis. A significant limitation to the use of AFP for HCC surveillance is the rate of AFP-negative HCC. Up to $50 \%$ of small HCCs do not secrete AFP and even with larger lesions, 20\% are not associated with elevated levels (Sherman et al., 2014). These results confirmed those of Zhu et al. (2013) who found that the sensitivity of MDK in AFP-negative HCC could reach as high as $89.2 \%$. The higher sensitivity in the Zhu et al study probably reflects the fact that they had a lower percentage of early stage tumours (49\%) and were from a single centre in China with mainly HBV-related HCC.

\section{CONCLUSION}

AFP and MDK may have a complementary role in HCC surveillance and screening. MDK increases the diagnostic yield in AFP-negative HCC and the presence of either elevated AFP or MDK increases the sensitivity of $\mathrm{HCC}$ detection. AFP is negative $(<20 \mathrm{IU} / \mathrm{ml})$ but MDK elevated ( $\geq 0.39 \mathrm{ng} / \mathrm{ml})$, a higher index of suspicion for an AFP-negative tumour is warranted. If initial ultrasound imaging is negative, these patients might require a shorter surveillance interval or more diagnostic modalities such as CT or MRI. MDK is also superior to AFP in the diagnosis of NASH-related HCC and this finding postulates an exciting novel role for MDK in NASH-related carcinogenesis that warrants further investigation.

\section{REFERENCES}

1. Aynacioğlu AŞ, Bilir A and Tuna MY. (2019): Involvement of midkine in autoimmune and autoinflammatory diseases. Modern Rheumatology, 29(4):567-71.

2. Bruix J, Gores GJ and Mazzaferro V. (2014): Hepatocellular carcinoma: clinical frontiers and perspectives. Gut, 63(5):844-55.

3. Chan AJ, Balderramo D, Kikuchi L, Ballerga EG, Prieto JE and Tapias $M$. (2017): Early age hepatocellular carcinoma associated with hepatitis B infection in South America. Clin Gastroenterol Hepatol., 15:1631-1632. 
4. Forner A, Reig $M$ and Bruix J. (2018): Hepatocellular carcinoma. Lancet, 391: 13011314.

5. Giannelli G, Rani B, Dituri F, Cao Y and Palasciano G. (2014): Moving towards personalised therapy in patients with hepatocellular carcinoma: the role of the microenvironment. Gut, 63(10):1668-76.

6. Hodeib H, ELshora O, Selim A, Sabry NM and EL-ashry HM. (2017): Serum midkine and osteopontin levels as diagnostic biomarkers of hepatocellular carcinoma. Electronic Physician, 9(1):3492-98.

7. Kadomatsu K, Kishida $S$ and Tsubota S. (2013): The heparin-binding growth factor midkine: the biological activities and candidate receptors. J Biochem., 153(6):511-21.

8. Mao Y, Yang H, Xu H, Lu X, Sang X, Du S, Zhao H, Chen W, Xu Y, Chi T and Yang $Z$. (2010): Golgi protein 73 (GOLPH2) is a valuable serum marker for hepatocellular carcinoma. Gut., 59(12):1687-93.

9. Mehinovic L, Islamagic E, Husic-Selimovic A, Kurtovic-Kozaric A, Vukobrat-Bijedic Z and Suljevic D. (2018): Evaluation of Diagnostic Efficiency of Alpha-Fetoprotein in Patients with Liver Cirrhosis and Hepatocellular Carcinoma: Single-Center Experience. Open Access Maced J Med Sci., 6(9): 1668-1673.

10. Meringer H, Shibolet $O$ and Deutsch $L$. (2019): Hepatocellular carcinoma in the posthepatitis $C$ virus era: Should we change the paradigm?. World Journal of Gastroenterology, 25(29):3929-34.

11. Moucari R, Asselah T, Cazals-Hatem D, Voitot H, Boyer N, Ripault MP, Sobesky R, Martinot-Peignoux M, Maylin S, NicolasChanoine MH and Paradis V. (2010): Insulin resistance in chronic hepatitis $\mathrm{C}$ : association with genotypes 1 and 4, serum HCV RNA level, and liver fibrosis. Gastroenterology, 134(2):416-23.
12. Muramatsu T and Kadomatsu K. (2014): Midkine: An emerging target of drug development for treatment of multiple diseases. Br J Pharmacol., 171(4):811-3

13. Shaheen KY, Abdel-Mageed AI, Safwat E and Ashraf M. (2015): The value of serum midkine level in diagnosis of hepatocellular carcinoma. Int J Hepatol., 2015:146389

14. Shaker MK, Abdella HM, Khalifa MO and Dorry AK. (2013): Epidemiological characteristics of hepatocellular carcinoma in Egypt: a retrospective analysis of 1313 cases. Liver International, 33(10):1601-6.

15. Sherman M, Furlan A, Marin D, Agnello F, Martino Di M and Marco Di V. (2014): Surveillance for hepatocellular carcinoma. Best Pract Res Clin Gastroenterol., 28:783-793.

16. Singhal A, Jayaraman M, Dhanasekaran DN and Kohli V. (2012): Molecular and serum markers in hepatocellular carcinoma: predictive tools for prognosis and recurrence. Crit Rev Oncol Hematol., 82:116-140.

17. Stefaniuk P, Cianciara J and WiercinskaDrapalo A. (2010): Present and future possibilities for early diagnosis of hepatocellular carcinoma. World Journal of Gastroenterology, 16(4):418-24.

18. Weckbach LT, Preissner KT and Deindl E. (2018): The role of Midkine in Arteriogenesis, involving Mechanosensing, endothelial cell proliferation, and vasodilation. International Journal of Molecular Sciences, 19(9):25592563.

19.Zhang BH, Li B, Kong LX, Yan LN and Yang JY. (2020): Diagnostic accuracy of midkine on hepatocellular carcinoma: A metaanalysis. PloS One, 14(10):e0223514.

20.Zhu WW, Guo JJ, Guo L, Jia HL, Zhu M, Zhang JB, Loffredo CA, Forgues M, Huang H, Xing XJ and Ren N. (2013): Evaluation of midkine as a diagnostic serum biomarker in hepatocellular carcinoma. Clinical Cancer Research, 19(14):3944-54. 


\section{قيمه مستوي الميلكين فى اللدم فى مرضى التليف الكبلي وسرطان الكبل}

أحمد ابراهيم السيد مندور, مجدي عبدالكريم الدهشان, عمر محمد خليل هيكل, محمد سعد الدين رضوان*, محمد شحات حسيب الفيومي مبدي

قسمي أمراض الباطنة, والباثولوجيا الاكلينيكية*, كلية الطب، جامعة الأزهر

E-mail: ahmedmandour24@gmail.com

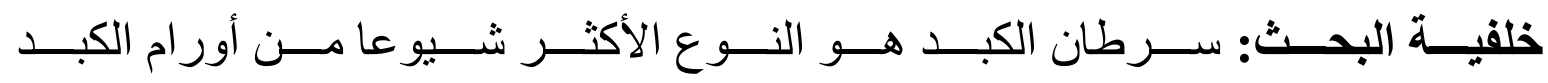

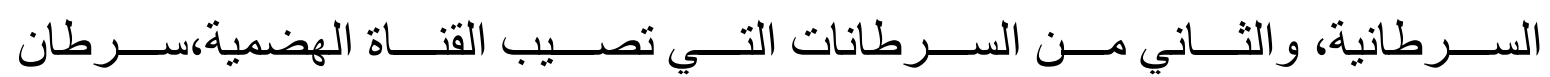

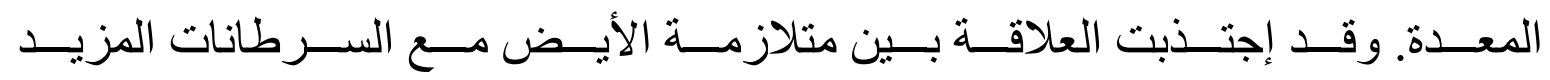

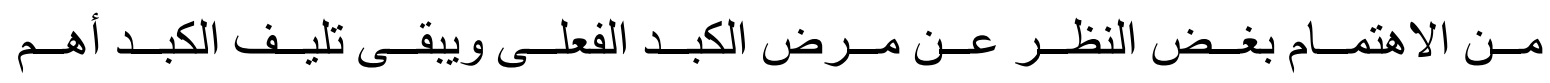

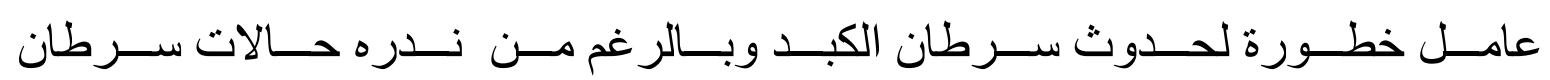

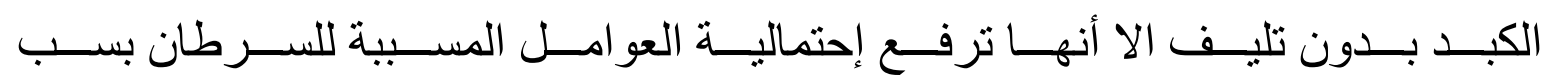

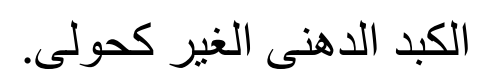

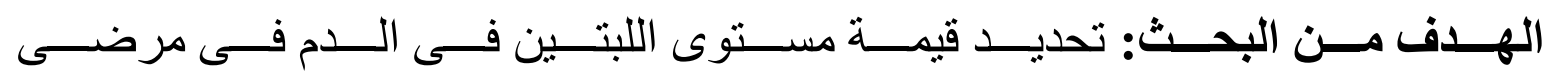

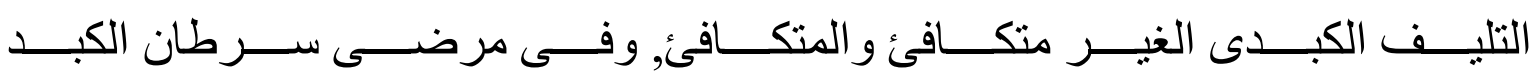
و علاقة هذا المستوى بالمعدل الأيضى فى هؤ لاء المرضى.

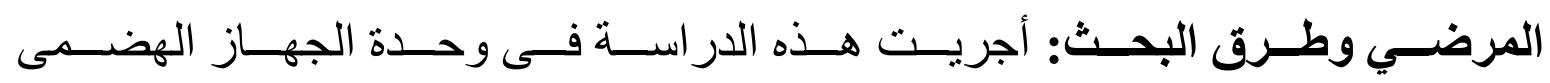

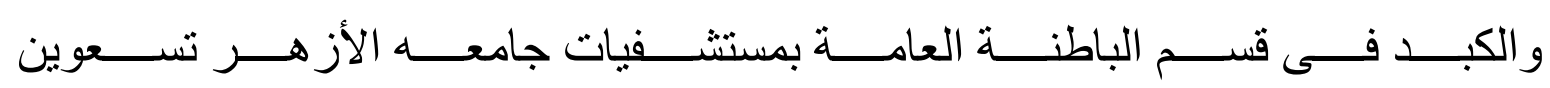

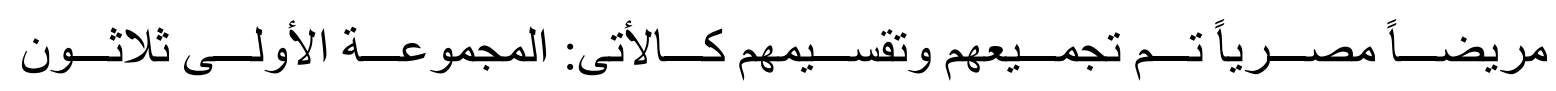

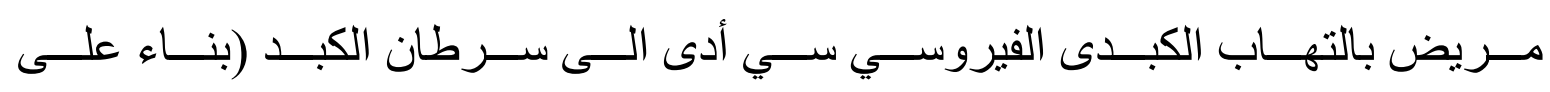

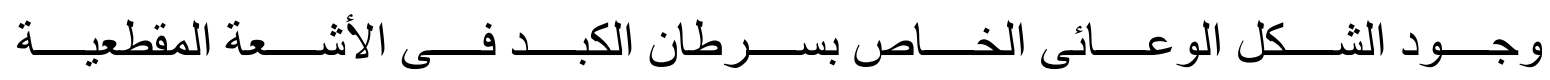

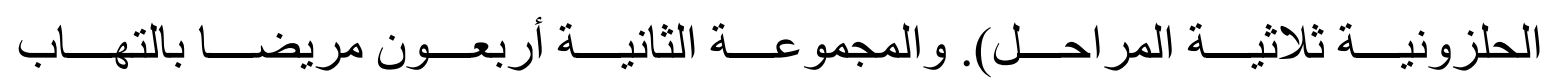

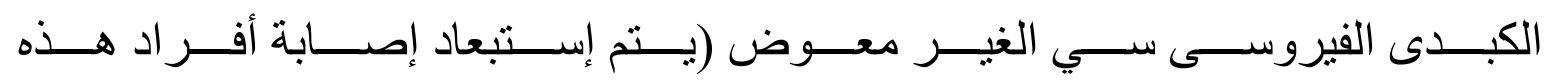

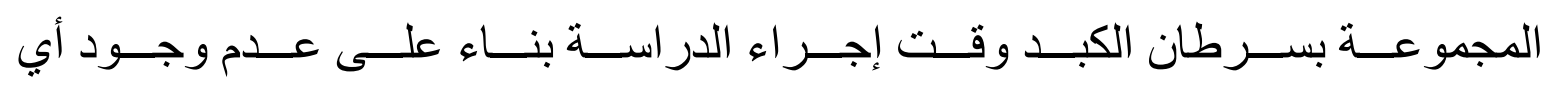




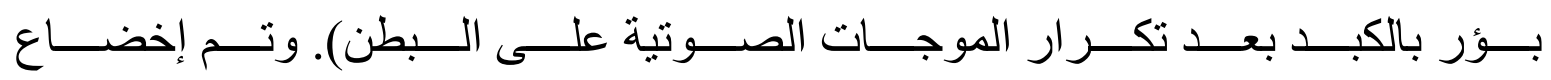

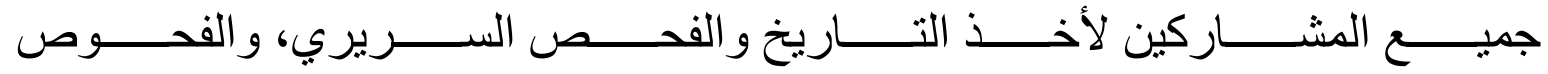

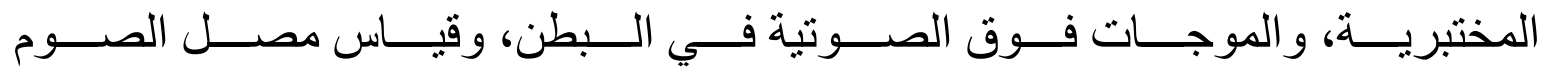
مستويات الأنسولين و اللبتين في الدم.

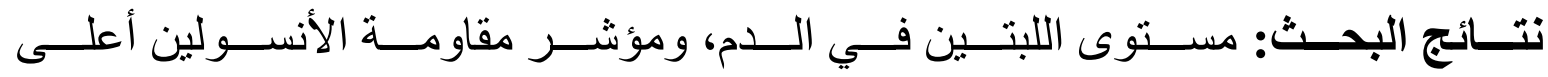

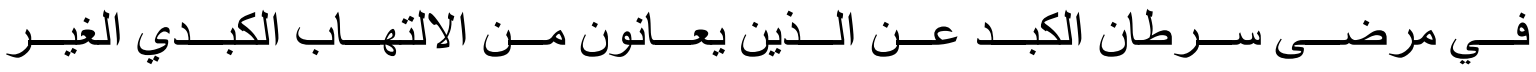

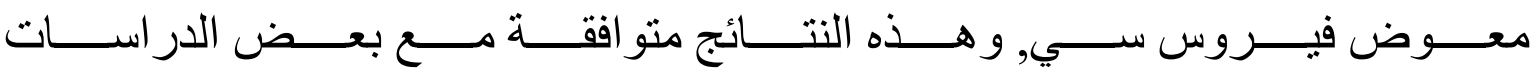
وإرتباطها بحدوث سرطان الكبد.

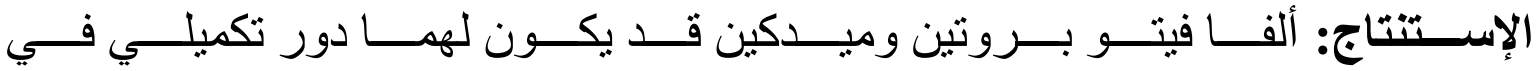
مر اقبة وفحص سرطان الخلايا الكبدية. الكلمات الدالة : ميدكين - التليف الكبدى - سرطان الكبد . 